\title{
High Rate Transmission in Spatial Modulation
}

\author{
Reshma John ${ }^{1}$, Niyas K. Haneefa ${ }^{2}$ \\ ${ }^{I}$ (ECE, Mar Baselios College of Engineering and Technology, India) \\ ${ }^{2}$ (ECE, Mar Baselios College of Engineering and Technology, India)
}

\begin{abstract}
Multiple Active Antenna -Spatial Modulation (MA-SM) is a high rate, low complexity MIMO transmission scheme. It takes the advantage of low complexity of SM and high multiplexing gain of V-BLAST system. Here, the information bits are conveyed by both the constellation symbol and spatial symbol. To benefit the available multiplexing gain, active antennas could be any number not more than the number of transmit antennas. However, this leads to degradation in system performance due to the presence of ICI and IAI. In this paper, SVD based precoding technique and Genetic Algorithm based symbol detection have been introduced to reduce ICI and thereby improving the system performance. Simulation results show that the proposed scheme improves the BER performance.
\end{abstract}

Keywords: Genetic Algorithm (GA), Generalised Spatial Modulation (GSM), Multiple Active Antenna Spatial Modulation (MA-SM), Multiple Input Multiple Output (MIMO) system, Maximum Likelihood (ML) detection.

\section{Introduction}

Wireless communication is the fastest growing segment of the communication industry. Many technical challenges remain in designing robust wireless networks that deliver performance necessary to support emerging applications. The signal from the transmitter will reach the receiver through a number of paths. This signal will undergo attenuation, distortions, delay and phase shifts. Both constructive and destructive interference will occur at the receiver. Destructive interference causes the signal to get diminished at the receiver. This phenomenon is called fading which causes deterioration of system quality. In order to guarantee a reliable communication, wireless system must be free from fading and interference. Fading can be mitigated by diversity. Several diversity techniques have been employed in wireless communication. Some of them are frequency diversity, time diversity, and space diversity. In frequency diversity technique, information signal is modulated through $\mathrm{M}$ different carriers. In time diversity, the desired signal is transmitted in $M$ different periods of time. In space diversity, we use $M$ antennas to receive $M$ copies of the transmitted signal. Among different space diversity techniques MIMO system have been theoretically shown to offer substantial increase in spectral efficiency compared to single antenna systems. Various MIMO systems have been designed to achieve either diversity gain or multiplexing gain, or even both. Diversity gain improves the coverage and QoS, whereas multiplexing gain will increases the spectral efficiency. Space Time Block Coding (STBC) and Spatial Multiplexing are the two MIMO transmission schemes that can achieve diversity gain and multiplexing gain respectively.

STBCs are used in MIMO systems to enable the transmission of multiple copies of a data stream. It combines all the copies of the received signal in an optimal way to extract as much as information from each of them as possible. Improvement in the performance is due to the use of both the temporal and spatial diversity. They are a generalized version of Alamouti's scheme. The data to be transmitted are in form of a matrix, in which column is equal to the number of transmit antenna and row equal to the number of time slot needed for transmission. A special category of STBCs are orthogonal STBCs (OSTBC) which offer full diversity with single symbol ML detection. Main disadvantage is that rate one full diversity OSTBC exists only for the case of 2 transmit antenna. For more than 2 transmit antenna, the maximum rate is upper bounded to $3 / 4$ symbols per channel use resulting in a loss in MIMO channel capacity. Another type of STBCs achieving full rate full diversity is Quasi-Orthogonal STBC (QO-STBC). However a big disadvantage of QO-STBC is that ML detection complexity grow exponentially with the number of transmit antennas and modulation order [1].

Spatial multiplexing is developed due to increase in demand for high rates in modern wireless communication systems. It doesn't require any bandwidth expansion. If fading process of spatial channels is independent, then an equalizer is used to separate the data streams. A well-known spatial multiplexing scheme is the Vertical Bell Layered Space Time (V-BLAST) system. In this system independent co-channel signals are transmitted simultaneously from transmit antennas, hence creating a high level of inter-channel interference (ICI) at the receiver. This phenomenon causes the complexity of optimal ML detector to grow exponentially with the number of transmit antennas. The use of suboptimal decoder, such as ZF or MMSE decoder allows low detection complexity with degradation in BER performance [2].

Spatial Modulation(SM) is an alternative to spatial multiplexing MIMO technique. SM enables high date rate transmission without ICI. In SM transmission schemes, the information data is conveyed by both the conventional amplitude/phase modulation technique and antenna indices. Here, only one antenna is activated at a

DOI: 10.9790/2834-1104049197 $\quad$ www.iosrjournals.org $91 \mid$ Page


time. Thus ICI is completely eliminated. Similar to V-BLAST systems, the SM system aims at exploiting the multiplexing gain of transmit antennas without taking the transmit diversity of MIMO system into consideration. The SM require the number of transmit antenna to be a power of 2 in order to achieve an increase of $\log _{2} \mathrm{n}_{\mathrm{T}}$ bits/s/Hz in spectral efficiency [2], [3].

Generalised Spatial Modulation (GSM) is a modification of SM technique. In GSM, the same symbol is transmitted simultaneously from more than one transmit antenna. In order to increase the data rate, GSM is again modified to Multiple Active Antenna Spatial Modulation (MA-SM). In MA-SM, different antennas will transmit different symbols, thus increasing the data rate. The active TA in MA-SM is not only a physical component that radiates electromagnetic wave, but also an information bearing source. Increase in the number of active antennas leads to an increase in the capacity of the system but leads to degradation in system performance due to ICI and IAI. In this paper, precoding techniques and genetic algorithm based symbol detection have been introduced in MA-SM in order to reduce the ICI.

\section{Multiple Active Antenna Spatial Modulation}

In Multiple Active Antenna Spatial Modulation (MA-SM), multiple active antennas will transmit multiple symbols. MA-SM maps the information bits into both spatial dimension and traditional complex dimension. The information is conveyed by both the modulated symbol and antenna indices. So the data rate of MA-SM will be more than GSM [2].

Consider a MIMO system with $\mathrm{N}_{\mathrm{T}}$ transmit antenna and $\mathrm{N}_{\mathrm{R}}$ receive antenna. An $\mathrm{M}$ order Quadrature Amplitude modulation (M-QAM) will map the information bits into $\mathrm{N}_{\mathrm{A}}$ active antennas. The transmitted symbol comprised of $N_{A}$ QAM symbols emitted from the corresponding active antennas. The active antenna is represented by set of 0 's and 1's, where 1 and 0 represent the on and off state of transmit antennas respectively. For $N_{T}$ transmit and $N_{A}$ active antennas there will be $N_{T} C N_{A}$ possible antenna groups. Consider a system with $N_{T}=4$ and $N_{A}=2$, the possible antenna groups are $\xi_{1}=(1,1,0,0), \xi_{2}=(1,0,1,0), \xi_{3}=(1,0,0,1), \xi_{4}=$ $(0,1,1,0), \xi_{5}=(0,1,0,1), \xi_{6}=(0,0,1,1)$. The received signal through $N_{T} \times N_{R}$ wireless channel $\mathrm{H}$ and $N_{R}$

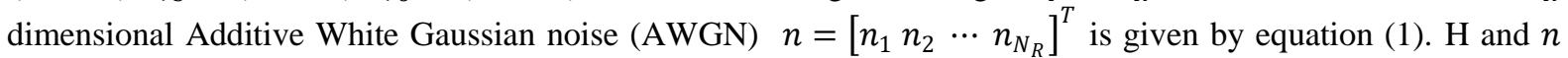
are independent and identically distributed (i.i.d) entries according to $C N(0,1)$.

$$
\begin{aligned}
& y=\sqrt{\frac{\rho}{N_{A}}} H^{H} X+n \\
& X \triangleq\left[\begin{array}{llll}
0 s_{1} \cdots s_{N_{A}} & 0
\end{array}\right]^{T}
\end{aligned}
$$

Where,

$X$ is the data vector of dimension $N_{T}$

$s_{1} \cdots s_{N_{A}} \in S$ is modulated symbol selected from the constellation set $\mathrm{S}$.

$a_{1}, \cdots, a_{N_{A}} \in\left\{1, \cdots, N_{A}\right\}$ is the antenna selected for the transmission of $s_{1} \cdots s_{N_{A}}$ symbols.

$\rho$ is the average signal to noise ratio (SNR) at each receive antenna [2].

\subsection{MA-SM transmitter}

(1) Select $N_{A}$ antennas from $N_{T}$ as antenna groups. For every $N_{T}$ transmit antenna and $N_{A}$ active antennas, there will be $2^{\left[\log _{2}\left(N_{T} C N_{A}\right)\right\rfloor}$ legal antenna groups [2].

(2) Encode the antenna groups into binary sequence of length $\left\lfloor\log _{2}\left(N_{T} C N_{A}\right)\right\rfloor$ [2].

(3) Divide the information bits into $N_{A} * \log _{2}\left(N_{T} C N_{A}\right)+\left[\log _{2}\left(N_{T} C N_{A}\right)\right\rfloor$ streams.

(4) Map the $N_{A} * \log _{2}\left(N_{T} C N_{A}\right)$ bits in each stream into QAM symbols.

(5) Last $\left[\log _{2}\left(N_{T} C N_{A}\right)\right\rfloor$ bits in each stream are used to determine the antenna group.

(6) Transmit the mapped $N_{A} * \log _{2}\left(N_{T} C N_{A}\right)$ symbol streams from the $N_{A}$ active antenna.

Table 1.1: MA-SM Transmit Symbol Mapping for $\mathrm{M}=4$

\begin{tabular}{|c|c|}
\hline \multicolumn{2}{|c|}{$N_{T}=4, N_{P}=2, M=4$} \\
\hline Input Bits & Transmit Symbol \\
\hline 00 & $-1+\mathrm{j}$ \\
\hline 01 & $-1-\mathrm{j}$ \\
\hline 10 & $1+\mathrm{j}$ \\
\hline 11 & $1-\mathrm{j}$ \\
\hline
\end{tabular}

Table 1.2: MA-SM Transmit Antenna Mapping for $N_{P}=2$

\begin{tabular}{|c|c|}
\hline \multicolumn{2}{|c|}{$N_{T}=4, N_{P}=2, M=4$} \\
\hline Input Bits & Antenna Number \\
\hline 00 & 1,4 \\
\hline
\end{tabular}




\begin{tabular}{|c|c|}
\hline 01 & 1,3 \\
\hline 10 & 2,4 \\
\hline 11 & 2,3 \\
\hline
\end{tabular}

\subsection{MA-SM receiver}

The received signal information contains both symbol information and antenna index information. Thus two set detection are available at the receiver. First is the antenna set detection and second symbol detection. Assuming the indices of the active antenna as $a_{1}, \cdots, a_{N_{A}}$, the signal transmission model can be rewritten as

$$
y=\sum_{j=1}^{N_{A}} h_{j} s_{j}+n[2]
$$

The antenna group is detected as

$$
\left(a_{1}, \ldots, a_{N_{A}}\right)=\arg \max _{k}\{y\}
$$

Now convert the row number to binary which will give the antenna that was used for transmission. After the antenna detection, the symbols undergo M-QAM demodulation. Both the demodulated symbol and antenna group are combined to regain the information bit.

The capacity of MA-SM system is given by

$$
C=N_{A} * \log _{2}\left(1+\frac{S N R}{N_{A}}\right)+\left\lfloor\log _{2}\left(N_{T} C N_{A}\right)\right\rfloor[2]
$$

\section{Proposed Scheme}

In MA-SM, $N_{A}$ can be any number less than the number of transmit antenna Consider a system with $N_{T}=4$ and $N_{A}=3$, the possible antenna groups are $\xi_{1}=(1,1,1,0), \xi_{2}=(1,1,0,1), \xi_{3}=(1,0,1,1), \xi_{4}=$ $(0,1,1,1)$. Increase in $N_{A}$ will leads to increase in multiplexing gain and ICI, which degrade the system performance. ICI can be eliminated by precoding at transmitter as well by using genetic algorithm for symbol detection at receiver.

Table 1.3: MA-SM Transmit Antenna Mapping for $N_{P}=3$

\begin{tabular}{|c|c|}
\hline \multicolumn{2}{|c|}{$N_{T}=4, N_{P}=3, M=4$} \\
\hline Input Bits & Antenna Number \\
\hline 00 & $2,3,4$ \\
\hline 01 & $1,2,3$ \\
\hline 10 & $1,3,4$ \\
\hline 11 & $1,2,4$ \\
\hline
\end{tabular}

\subsection{Precoding Scheme}

Precoding is a processing technique which exploits the channel state information at transmitter (CSIT) by operating signals before transmission. Designing a system with channel knowledge at the transmitter will increases the capacity compared to the system with channel knowledge at receiver. The usage of transmitter power can be handled more intelligently using the precoder matrix thus increasing the efficiency and quality of the transmission [4]. SVD based precoder has been chosen that will maximize the minimum received signal. It is an optimal way to extract spatial multiplexing gains in MIMO channels. It is a composition of three operations: a rotation, a scaling operation and another rotation. It can be represented as a product of a unitary matrix and a diagonal matrix.

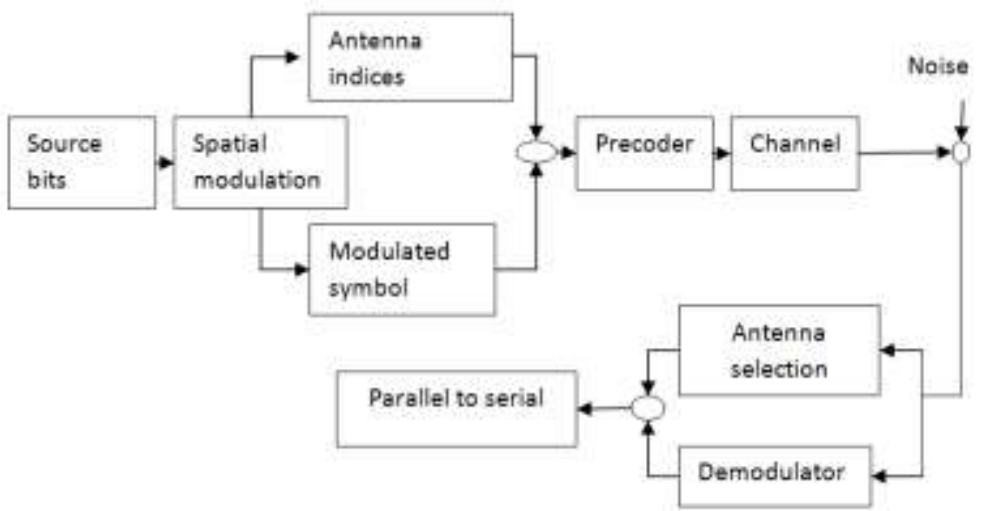

Fig 1: SYSTEM MODEL 
The received signal through $N_{T} \times N_{R}$ wireless channel $\mathrm{H}$ and $N_{R}$ dimensional Additive White Gaussian noise (AWGN) $n=\left[\begin{array}{llll}n_{1} & n_{2} & \cdots & n_{N_{R}}\end{array}\right]^{T}$ after precoding is given by

Where,

$$
y=\sqrt{\frac{\rho}{N_{A}}} H^{H} P X+n[5]
$$

$\mathrm{P}$ is the $N_{T} \times N_{T}$ precoded matrix.

$X$ is the data vector of dimension $N_{T}$.

$\rho$ is the average signal to noise ratio (SNR) at each receive antenna.

$\mathrm{H}$ and $n$ are independent and identically distributed (i.i.d) entries according to $C N(0,1)$.

The precoded matrix is obtained by the SVD decomposition of channel matrix i.e., $H=U S V^{H}$ where

$U$ and $V^{H}$ are unitary matrices and $\mathrm{S}$ is a diagonal matrix with diagonal entries equal to the eigen values of the channel. The eigen vectors of $H * H^{H}$ forms the column of $\mathrm{U}$ and the eigen vectors of $H^{H} * H$ forms the column of V.S is the square root of eigen values from $H * H^{H}$ and $H^{H} * H$. Independent orthogonal sub channels can be defined according to the orthogonal eigenvectors of the channel that have a capacity proportional to the corresponding eigen values. Here antennas have been weighted according to the eigen values of the channel. The precoded matrix $\mathrm{P}$ is given as

$$
P=V
$$

The transmission scheme can be optimized by maximizing the minimum distance between the code words that dominates BEP (Bit Error Probability) [2].

At the receiver, prior to symbol and antenna detection the received symbol is multiplied with $U^{H}$. Assuming the indices of the active antenna $a_{1}, \cdots, a_{N_{A}}$, the signal transmission model can be rewritten as

The antenna group is detected as

$$
y=\sum_{j=1}^{N_{A}} h_{j} V_{j}^{H} s_{j}+n
$$

$$
\left(a_{1}, \ldots, a_{N_{A}}\right)=\arg \max _{k}\left\{U^{H} y\right\}
$$

Now convert the row number to binary which will give the antenna that was used for transmission. After the antenna detection, the symbols undergo M-QAM demodulation. Both the demodulated symbol and antenna group are combined to regain the information bit.

\subsection{GA BASED SYMBOL DETECTION}

GA presumes that the potential solution of any problem is an individual and can be represented by a set of parameters regarded as the genes of a chromosome and can be structured by a string of values in binary form. Genetic algorithm mainly consists of individual, population and a fitness function. Fitness function is the objective function to be minimized. Population is a set of all possible solution of problem. Individual is each element of population. Genetic algorithm uses a set, or population, of points to conduct a search, not just a single point on the problem space. This gives GAs the power to search noisy spaces littered with local optimum points [6].

The simplest form of genetic algorithm involves three types of operators: selection, cross over and mutation.

- Initialization

All potential solutions of a problem are encoded in bit level to simplify the following GA recombination operation. At first, initialization of population is done and output from linear/non-linear detector is derived. Each chromosomes is a combination of probable solution for all the transmit antennas.

- Selection

Selection is based on the fitness function. Population is evaluated for each generation and fitness value. In a generation, the best chromosomes should have the best fitness function. Each generation value is compared with the previous values of fitness function and the least value is chosen as the best solution.

- Reproduction

Reproduction makes extra copies of improved strings in a new population. Reproduction is usually the first operator applied on a population. Reproduction selects good strings in a population and also replaces the largest value of fitness function with the best solution. It contains two operator namely, crossover and mutation.

A crossover operator is used to recombine two strings to get an improved string. In crossover operation, recombination process creates different individuals in the successive generations by combining material from two individuals of the previous generation. In crossover, new strings are created by exchanging information between the parent node which are selected randomly and creates offspring.

Mutation simply changes each bit of the randomly selected binary chromosome. It individually replaces the worst fitness value by the best solution. In mutation probability, the bit is inverted, so that zero becomes one and one becomes zero. This helps in introducing a bit of diversity to the population by scattering the occasional points. This random scattering would result in the better optimal, or even modify a part of genetic code that will be beneficial in later operations. 
- Termination

Generation process is repeated until a termination condition has been reached. This can be when the fixed number of generations has been reached or the allocated level has reached.

Algorithm works as follows:

- Generate the random initial population which contains the possible symbols transmitted. Each solution in a population is known as individual.

- Evaluate the objective function for each individual.

The objective function is given as

Where, $\hat{X}$ is the probable solution for all transmit antenna.

$$
f=\|H \hat{X}\|
$$

- Compare the objective function with the fitness function, $\|Y\|$.

- If fitness function is greater than the objective function, new population is created and selection process is repeated else the corresponding symbol is selected.

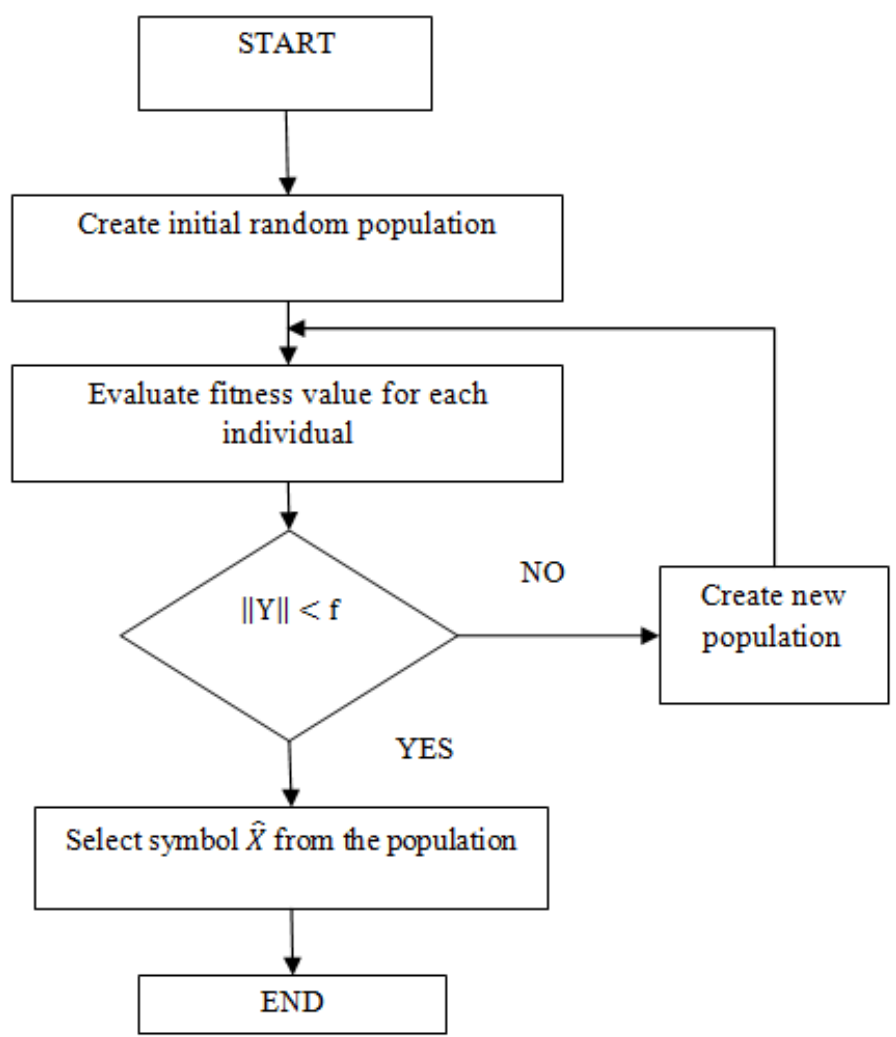

Fig 2: Flow chart of Genetic Algorithm for Symbol Detection

After symbol selection antenna is detected from the selected symbol. The number of population gives the number of active transmit antenna. The antenna group is detected as

$$
\left(a_{1}, \ldots, a_{N_{A}}\right)=\arg \max _{k}\{\|\hat{X}\|\}
$$

Now convert the row number to binary which will give the antenna that was used for transmission. The symbols undergo M-QAM demodulation. Both the demodulated symbol and antenna group are combined to regain the information bit.

\section{Simulation Results}

In this section, we provide some simulation results for MA-SM. MA-SM is compared with the other MIMO schemes such as Alamouti's scheme and V-Blast. Fig 3 gives the comparison of MA-SM with the Alamouti's scheme and V-Blast at 6 Bits/s/Hz. Fig 4 gives the Capacity of MA-SM with $N_{A}=2$ and $N_{A}=3$. This shows that there is an increase in the capacity of the system with $N_{A}=3$. Fig 5 gives the BER performance of MA-SM with $N_{A}=2$ and $N_{A}=3$. It can be seen that there is degradation in the BER performance with increase in the number of active antennas. Fig 6 gives the BER performance of MA-SM after introducing precoding. It can be seen that there is an improvement in system performance with precoding. Fig 7 gives the BER performance of MA-SM with GA based detection. 


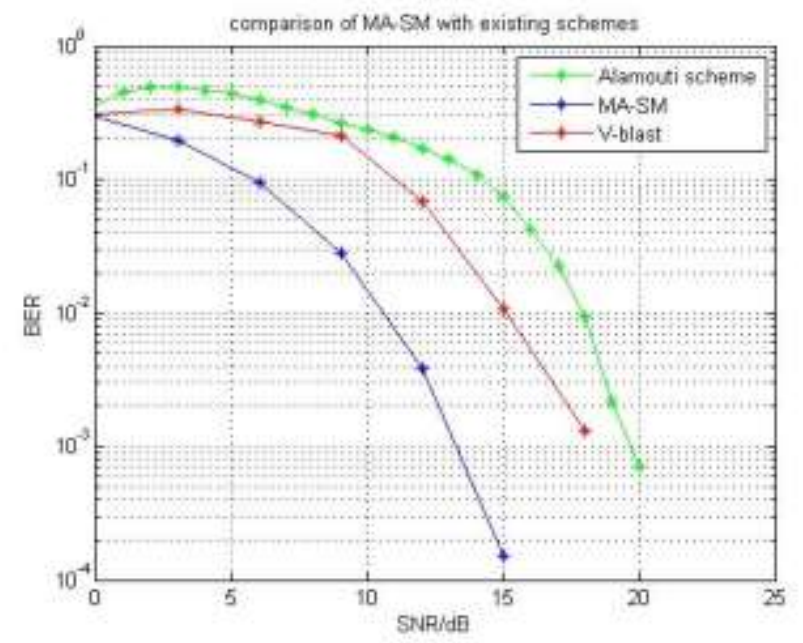

Fig 3: Comparison of MA-SM with Alamouti's scheme and V-Blast

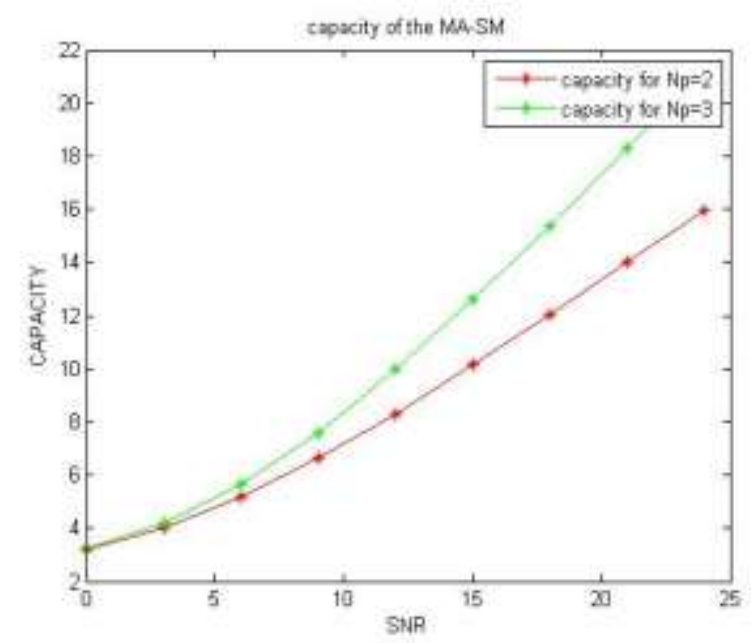

Fig 4: Capacity of MA-SM with different active transmit antenna.

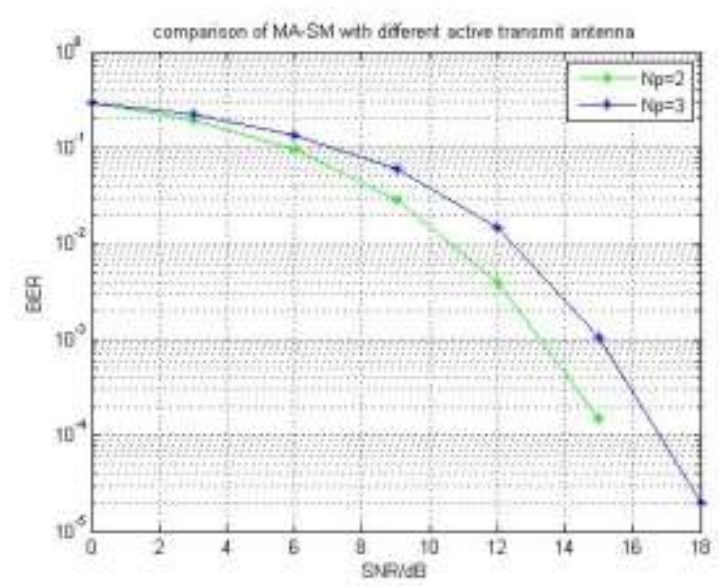

Fig 5: BER performances of MA-SM with different number of active transmit antennas. 


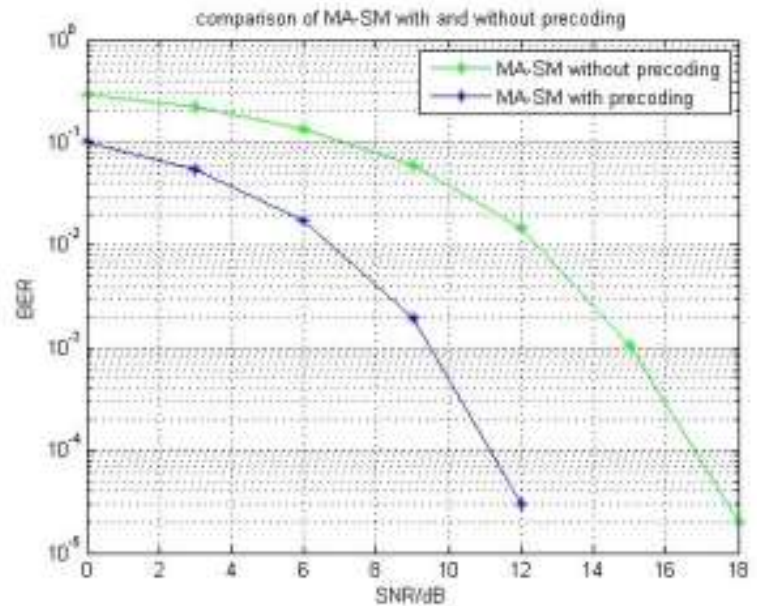

Fig 6: BER performance of MA-SM with and without precoding

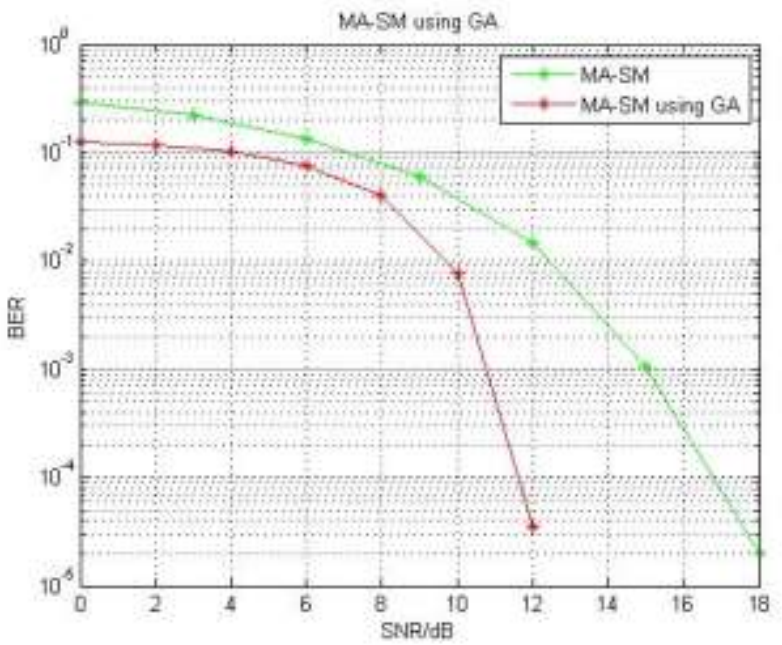

Fig 7: BER performance of MA-SM with different detection scheme

\section{Conclusion}

Multiple Active Antenna Spatial Modulation have been introduced in this paper as an alternative for the MIMO schemes such as Alamouti's scheme and V-Blast. In MA-SM, multiplexing gain can be improved by increasing the number of active transmit antenna but it causes a degradation in system performance. Here, we have introduced a precoding technique and a GA based symbol detection method to improve the performance of the system. It has been shown via computer simulation that MA-SM system offers significant improvement in system performance after introducing precoding.

\section{References}

[1]. E. Basar, U. Aygolu, E. Panayirci, and H. V. Poor, "Space-time block coded spatial modulation", IEEE Trans. Communication., vol. 59, no. 3, pp. 823-832, Mar. 2011.

[2]. Jintao Wang, Shuyun Jia, Jian Song "Generalized spatial modulation system with multiple active transmit antennas and low complexity detection schemes", IEEE Trans.on Wireless communication, vol. 11, no. 4, April 2012.

[3]. Faseela K. Karim, Basil K. Jeemon, "Spatial Modulation with Multiple Active Transmit Antennas", IJMTER, vol. 2, Issue 11, ISSN: 2393-8161, Nov 2015.

[4]. Ambuj Mehrish, Vikash Kumar, Ashish Goswami, "Precoding based on signal-to leakage and noise ratio to reduce ICI in MIMOOFDM systems", International Journal of Computer Application, vol. 95, no. 19, June 2014.

[5]. Ganesan Thiagarajan, Chandra R. Murthy, "Novel transmit precoding methods for Rayleigh fading multiuser TDD-MIMO systems with CSIT and no CSIR", IEEE Trans. on Vehicular technology, vol. 64, 2015.

[6]. Nazmat Surajudeen-Bakinde, Xu Zhu, Jingbo Gao , Asoke K. Nandi, ” Improved Signal Detection Approach using Genetic Algorithm for overloaded MIMO systems", $4^{\text {th }}$ International Conference on wireless communications, networking and Mobile Computing, Oct 2008 